\title{
THE ROLE OF MINIMAL IDEMPOTENTS IN THE REPRESENTATION THEORY OF LOCALLY COMPACT GROUPS
}

\author{
by BRUCE A. BARNES
}

(Received 16th May 1979)

\section{Introduction}

In the representation theory of finite groups, the minimal idempotents of the group algebra play a central role. In this case the minimal idempotents determine irreducible modules over the group algebra, which in turn are in direct correspondence with the irreducible matrix representations of the group; see Chapter IV of the book of C. Curtis and I. Reiner (2). Many of the same ideas generalise to the situation where the group is compact. In addition, minimal idempotents are involved in some important parts of the theory of Hilbert algebras; see M. Rieffel's paper (20).

For an arbitrary locally compact (LC) group $G$ there has been no exposition concerning the role that minimal idempotents of $L^{1}(G)$ or of $C^{*}(G)$ (the group $C^{*}$-algebra of $G$ ) play in the representation theory of $G$. In fact, it turns out that minimal idempotents of these algebras are intimately related to important (but special) facets of the representation theory of $G$ : square integrable representations, integral representations, and isolated points of $\hat{G}$, the dual of $G$.

In this paper we explore the part that minimal idempotents play in the representation theory of a general $L C$ group. A minimal idempotent of $C^{*}(G)$ determines an irreducible (strongly continuous unitary) representation of $G$. The equivalence class of this representation is an isolated point in $\hat{G}$. At least when $G$ is $\sigma$-compact, the converse is also true, an isolated point of $\hat{G}$ corresponds to an irreducible representation of $G$ determined by a minimal idempotent of $C^{*}(G)$ [Proposition 2]. When $G$ is unimodular and the minimal idempotent involved is in the reduced $C^{*}$-algebra of $G, C_{\rho}^{*}(G)$, then the corresponding irreducible representation is square integrable [Proposition 2]. If the minimal idempotent involved is in $L^{1}(G)$, then the corresponding representation is integrable [Theorem 1]. A simple corollary of this last result is that the equivalence class of an integrable representation of $G$ is open in $\hat{G}$ [Corollary 2]. This provides an elementary proof of the answer to an old question of J. Dixmier (4), previously answered by P. S. Wang in (15). As a further application of these ideas, using techniques involving minimal idempoten ts we prove that certain $L C$ groups (nilpotent Lie groups, Hermitian $I N$-groups) have the property that every irreducible square integrable representation is an integrable represntation [Theorem 3].

Notation and terminology: An $A^{*}$-algebra is a Banach *-algebra which has a faithful ${ }^{*}$-representation on Hilbert space (13, pp. 186-189). The algebra $L^{1}(G)$ or any $C^{*}$ - 
algebra are examples of $A^{*}$-algebras. Let $\boldsymbol{A}$ be an $\boldsymbol{A}^{*}$-algebra. An idempotent $\boldsymbol{e} \in \boldsymbol{A}$ is minimal if $A e$ is a minimal left ideal of $A$. This is equivalent to the property that for every $f \in A$, there exists a scalar $\lambda$ such that $e f e=\lambda e$. We usually deal with idempotents $e$ which both minimal and self-adjoint, $e=e^{*}$. Such idempotents determine irreducible *-representations of $A[\$ 2]$.

A representation of $A$ (or $G$ ) will always mean a *-representation of $A$ (a strongly continuous unitary representation of $G$ ) on Hilbert space. Representations are often denoted by a pair $(\pi, H)$ where $\pi$ is the map on $A$ (or $G$ ), and $H$ is the representation space.

$C^{*}(G)$ is the group $C^{*}$-algebra of $G\left(3, p^{-} .270\right)$. There is a one-to-one correspondence between representations of $G, L^{1}(G)$, and $C^{*}(G)$. We use the same Greek letter to denote the corresponding representation of all three. Let $\rho$ be the left regular representation of $G$ (or $L^{1}(G)$, or $C^{*}(G)$ ) on $L^{2}(G)$. Then $C_{p}^{*}(G)$ denotes the completion of $L^{1}(G)$ in the operator norm

$$
\|\rho(f)\|=\sup \left\{\|\rho(f) g\|_{2} ; g \in L^{2}(G),\|g\|_{2}=1\right\}, \quad f \in L^{1}(G) .
$$

Let $\operatorname{Irr}(G)(\operatorname{Irr}(A))$ be the collection of all irreducible representations of $G(A)$. The notation $\approx$ denotes unitary equivalence of representations. Let $\hat{G}(\hat{A})$ be the set of all equivalence classes of $\operatorname{Irr}(G)(\operatorname{Irr}(A))$ endowed with the Fell-topology (3, Definition, p. 60). As usual $\hat{G}$ is identified with $C^{*}(G)^{-}$and $\hat{G}_{r}$ with $C_{p}^{*}(G)^{-}$.

\section{Minimal idempotents and representation theory}

Throughout this section $A$ is an $A^{*}$-algebra, and $\mathscr{M}(A)$ is the set of all self-adjoint minimal idempotents of $A$. An idempotent $e \in \mathcal{M}(A)$ determines an irreducible *-representation of $A$ as described in C. Rickart's book (13, pp. 261-262). We proceed to briefly describe the construction of this representation and some of its basic properties. For every $f \in A$ there exists a complex number $\lambda_{f}$ such that $e f e=\lambda_{f} e$. Let $\alpha_{e}$ be the linear functional on $A$ defined by $\alpha_{e}(f)=\lambda_{f}$. Then $\alpha_{e}$ is a positive functional, and $\alpha_{e}$ determines an inner product on the minimal left ideal $A e$ by the rule

$$
\langle f e, g e\rangle=\alpha_{e}\left(e g^{*} f e\right) \quad(g, f \in A) .
$$

Now define a ${ }^{*}$-representation $\delta_{e}$ of $A$ on this inner product space by letting

$$
\delta_{e}(f)(g e)=f g e \quad(f, g \in A) .
$$

Denote the completion of the inner product space by $H_{e}$. Then $\delta_{e}$ extends to an irreducible ${ }^{*}$-representation of $A$ on $H_{e}$. We denote this extension also by $\delta_{e}$, and refer to $\left(\delta_{e}, H_{e}\right)$ as the irreducible ${ }^{*}$-representation of $A$ determined by $e$. Note that $\delta_{e}(e)$ is a projection of rank one on $H_{e}$. Thus $\delta_{e}(A)$ contains a non-zero operator with finite dimensional range, so that $\delta_{e}$ is FDS (finite dimensionally spanned; see (17, p. 231)).

Let $(\pi, H)$ be a *-representation of $A$, and assume that $\pi(e) \neq 0$. Fix $\xi \in H$ with $\pi(e) \xi=\xi$ and $\|\xi\|=1$. Let $K$ be the closure of $\pi(A) \xi$ in H. Define $U: A e \rightarrow K$ by

$$
U(f e)=\pi(f e) \xi=\pi(f) \xi \quad(f \in A) .
$$


Then for all $f e \in A e$

$$
\begin{aligned}
(U(f e), U(f e)) & =\left(\pi\left(e f^{*} f e\right) \xi, \xi\right) \\
& =\alpha_{e}\left(e f^{*} f e\right)(\pi(e) \xi, \xi) \\
& =\langle f e, f e\rangle .
\end{aligned}
$$

Then $U$ extends to a unitary map of $H_{e}$ onto $K$, and it is easy to check that this extension intertwines $\left(\delta_{e}, H_{e}\right)$ with the restriction of $\pi$ to $K$. We summarise these remarks as a proposition.

Proposition 1. Assume $e \in \mathcal{M}(A)$. If $(\pi, H)$ is a ${ }^{*}$-representation of $A$ such that $\pi(e) \neq 0$, then $\delta_{e}$, the irreducible ${ }^{*}$-representation of A determined by $e$, is unitarily equivalent to an irreducible subrepresentation of $\pi$. In particular, if $\pi$ is irreducible, then $\pi \approx \delta_{e}$.

Let $A$ be a $C^{*}$-algebra. If $\pi \in \operatorname{Irr}(A)$, then $\{\pi\}$ denotes the equivalence class of $\pi$ in $\hat{A}$. Fix $e \in \mathcal{M}(A)$. If $\pi \in \operatorname{Irr}(\mathrm{A})$, then by Proposition $1, \pi \neq \delta_{e}$ if and only if $\pi(e)=0$. Therefore

$$
e \in \cap\left\{\operatorname{ker}(\pi): \pi \in \operatorname{Irr}(A), \pi \neq \delta_{e}\right\} \text {. }
$$

Thus ker $\left(\delta_{e}\right)$ does not contain this intersection. This means in terms of the Fell-topology that $\left\{\delta_{e}\right\}$ is not in the closure of

$$
\left\{\{\pi\} \in \hat{A} ;\{\pi\} \not\left\{\delta_{e}\right\}\right\}
$$

or equivalently, that $\left\{\delta_{e}\right\}$ is open in $\hat{A}$. Conversely, assume that $A$ is separable (or more generally, that every irreducible *-representation of $A$ is separable). Suppose that $\{\pi\}$ is open in $\hat{A}$. Set

$$
M=\cap\{\operatorname{ker}(\tau) ;\{\tau\} \in \hat{A} \backslash\{\pi\}\} .
$$

Since $\{\pi\}$ is open, $M$ is nonzero. Then $M$ is a closed ideal of $A$ with $M \cap \operatorname{ker}(\pi)=\{0\}$. Using (3, Prop. 2.10.4) this implies that $\hat{M}$ consists of one element (the equivalence class of the representation $\pi$ restricted to $M)$. By (16, Lemma 1.3) $M$ is isomorphic to the algebra of completely continuous operators on some Hilbert space $H$. Let $e \in M$ correspond via this isomorphism to a self-adjoint projection on $H$ of rank one. Then it is straightforward to verify that $e \in \mathcal{M}(A)$. Also since $\pi(e) \neq 0$, Proposition 1 implies that $\pi \approx \delta_{e}$.

Now we translate the results above into the context of the representation theory of group algebras, taking $A=C^{*}(G)$ or $A=C_{\rho}^{*}(G)$. In these cases $\hat{A}$ is identified with $\hat{G}$ (the dual of $G$ ) and $\hat{G}_{r}$ (the reduced dual of $G$ ), respectively.

\section{Proposition 2. Let $G$ be a LC group. Then}

(1) if $e \in M\left(C^{*}(G)\right)$, then $\left\{\delta_{e}\right\}$ is open in $\hat{G}$;

(2) when $G$ is $\sigma$-compact if $\{\pi\}$ is open in $\hat{G}$, then there exists $e \in \mathcal{M}\left(C^{*}(G)\right)$ such that $\pi \approx \delta_{e}$;

(3) if $\in \in \mathcal{M}\left(C_{\rho}^{*}(G)\right)$, then $\left\{\delta_{e}\right\}$ is open in $G_{r}$; in this case, provided that $G$ is unimodular, $\delta_{e}$ is square integrable, and thus, $\left\{\delta_{e}\right\}$ is also closed in $\hat{G}(3$, Prop. 18.4.1);

(4) when $G$ is $\sigma$-compact, if $\{\pi\}$ is open in $G_{n}$ then there exists $e \in \mathcal{M}\left(C_{\rho}^{*}(G)\right)$ such that $\pi \approx \delta_{e}$.

The main part of the proof of Proposition 2 is contained in the remarks above. Note in 
this regard that every irreducible representation of a $\sigma$-compact group is separable. This fact and other pertinent results can be found in (1). Now we verify the statement in (3) that for $e \in M\left(C_{\rho}^{*}(G)\right), \delta_{e}$ is square integrable. Let $\bar{\rho}$ be the identity representation of $C_{\rho}^{*}(G)$ on $L^{2}(G)$. Then $\bar{\rho}(e) \neq 0$, so by Proposition $1 \delta_{e}$ is unitarily equivalent to a subrepresentation of $\bar{\rho}$. It follows that the corresponding group representation of $G$ is unitarily equivalent to some subrepresentation of the left regular representation of $G$ on $L^{2}(G)$.

Combining (3) and (4), we get the following result (compare with (4, Proposition 3)).

Corollary 1. Let $G$ be a $\sigma$-compact unimodular LC group. If $\{\pi\}$ is open in $\hat{G}_{m}$ then $\pi$ is square integrable.

Remarks. Does the converse of Corollary 1 hold, that is, if $\pi$ is square integrable, then is $\{\pi\}$ open in $\hat{G}_{r}$ ? This is an open question of J. Dixmier (4). Partial results have been obtained by T. Sund (14) and R. Lipsman (7).

It can happen that $\{\pi\}$ is open in $\hat{G}$, but $\pi$ is not square integrable. Examples are provided by noncompact groups with property $(T)(\mathbf{1 6}, \S 2)$. In this case the one dimensional identity representation of $G$ is open in $\hat{G}$ (by definition of property $(T)$ ), but this representation is not square integrable ( $G$ being noncompact).

\section{Integrable representations}

In this section we establish a basic connection between integrable representations of $G$ and irreducible representations of $L^{1}(G)$ determined by self-adjoint minimal idempotents of $L^{1}(G)$. Specifically we prove the following result.

Theorem 1. Let $G$ be a unimodular LC group. For $\pi \in \operatorname{Irr}\left(L^{\prime}(G)\right)$, the following are equivalent:

(1) $\pi$ is an integrable representation;

(2) $\pi$ is determined by some minimal idempotent in $M\left(L^{1}(G)\right)$;

(3) $\pi$ is FDS and Ann $(\operatorname{ker}(\pi)) \neq\{0\}$, where Ann $(\operatorname{ker}(\pi))=\left\{f \in L^{1}(G): f^{*} g=0\right.$ for all $g \in \operatorname{ker}(\pi)\}$.

We use Dixmier's notion of integrable representation (3, Definition 14.5.2). It is understood that an integrable representation is irreducible.

Before beginning the proof of Theorem 1, we note an interesting corollary of it.

Corollary 2. Let $G$ be a unimodular $L C$ group. If $\pi \in \operatorname{Irr}\left(L^{1}(G)\right)$ is integrable, then $\{\pi\}$ is open in $\hat{G}$.

The corollary follows immediately from Theorem 1 and Proposition 2 (1). It provides an answer to a question of J. Dixmier (4). This question has also been answered by S. P. Wang in (15) using very different methods.

We prove Theorem 1 by establishing a sequence of lemmas. Throughout $G$ is a $L C$ unimodular group.

We use the notation

$$
g_{x}(y)=g(x y) \text { where } x, y \in G \text { and } g \in L^{2}(G) .
$$


Lemma 1. Let $\pi$ be an integrable representation of $G$. Then $\pi$ is determined by some $e \in \mathcal{M}\left(L^{\prime}(G)\right)$.

Proof. Since $\pi$ is integrable, $\pi$ is square integrable, and we may assume that $\pi$ is an irreducible subrepresentation of $\rho$ (3, Lemma 14.1.1). Accordingly, let $M$ be the corresponding minimal left invariant subspace of $L^{1}(G)$. Then

$$
\pi(x) g=g_{x^{-1}} \in M \text { for all } g \in M \text { and all } x \in G
$$

Fix $\xi \in M, \xi \neq 0$, such that

$$
\phi(x)=(\pi(x) \xi, \xi) \in L^{1}(G) .
$$

We prove that some scalar multiple of $\phi$ is in $\mathcal{M}\left(L^{1}(G)\right)$. Note that if $f \in L^{1}(G)$ and $\eta \in M$ then $f * \eta \in M$ (in fact, $\pi(f) \eta=f * \eta)$.

For any $f \in L^{1}(G)$,

$$
\begin{aligned}
f * \phi(x) & =\int f(x y)\left(\pi\left(y^{-1}\right) \xi, \xi\right) d y \\
& =\overline{\int \bar{f}_{x}(y)(\pi(y) \xi, \xi) d y} \\
& =\overline{\left(\left(\bar{f}_{x}\right) * \xi, \xi\right)} \\
& =\overline{\left((\bar{f} * \xi)_{x}, \xi\right)} \\
& \left.=\overline{\left(\pi\left(x^{-1}\right) \eta, \xi\right)} \quad \text { (where } \eta=\bar{f} * \xi \in M\right) \\
& =(\pi(x) \xi, \eta) .
\end{aligned}
$$

For $\tau, \eta \in M$ we use the notation

$$
\phi_{\tau, \eta}(x)=(\pi(x) \tau, \eta) \quad(x \in G)
$$

In this notation we have shown that for any $f \in L^{1}(G)$,

$$
f * \phi=\phi_{\xi, \eta} \quad \text { where } \eta=\bar{f} * \xi \text {. }
$$

By (3, p. 278) there exists a number $d_{\pi}>0$ such that for any $f \in L^{1}(G)$ and $\eta$ as above

$$
\phi * f * \phi=\phi^{*} \phi_{\xi, \eta}=d_{\pi}^{-1}(\xi, \eta) \phi .
$$

It follows that $\phi$ is a scalar multiple of some element in $e \in \mathcal{M}\left(L^{1}(G)\right)$. Therefore also $\bar{e} \in \mathcal{M}\left(L^{1}(G)\right)$.

Now $\rho$ restricted to $J=L^{2}(G) * \vec{\phi}$ is an irreducible subrepresentation of $\rho$. Since $\rho(\bar{e})(J) \neq\{0\}$, by Proposition 1 this representation is determined by $\bar{e}$. Computing the positive definite function $(\rho(x) \bar{\phi}, \bar{\phi})$ we have

$$
\begin{aligned}
(\rho(x) \bar{\phi}, \bar{\phi}) & =\int \bar{\phi}\left(x^{-1} y\right) \phi(y) d y=\int \bar{\phi}\left(x^{-1} y\right) \bar{\phi}\left(y^{-1}\right) d y \\
& =\lambda \bar{\phi}\left(x^{-1}\right)=\lambda \phi(x)=\lambda(\pi(x) \xi, \xi)
\end{aligned}
$$

for some nonzero scalar $\lambda$. Therefore $\rho$ restricted to $J$ is equivalent to $\pi$ by $(3$, Theorem 13.4.5). Thus $\pi$ is determined by $\bar{e}$. 
Lemma 2. Assume that $e \in \mathcal{M}\left(L^{1}(G)\right)$. Then the representation determined by $e$ is integrable.

Proof. Note that $e \in L^{1} \cap L^{2}$ since for some $g \in L^{2}(G)$,

$$
e=e * g * e \in L^{2}(G)
$$

Now let $J=L^{2}(G) * e$. Then $J$ is a minimal closed left invariant subspace of $L^{2}(G)$ (because $\left.e \in \mathcal{M}\left(L^{1}(G)\right)\right)$. Furthermore $\rho$ restricted to $J$ is integrable since

$$
\begin{aligned}
(\rho(x) e, e) & =\int e\left(x^{-1} y\right) \overline{e(y)} d y \\
& =\int e\left(x^{-1} y\right) e\left(y^{-1}\right) d y \\
& =e * e\left(x^{-1}\right)=e\left(x^{-1}\right) \\
& =\overline{e(x)} \in L^{1}(G) .
\end{aligned}
$$

Since $e * J \neq\{0\}$, the representation determined by $e$ is equivalent to the integrable representation $\rho$ restricted to $J$.

Lemma 3. Let $A$ be an $A^{*}$-algebra. Assume $(\pi, H) \in \operatorname{Irr}(A)$. Then $\pi$ is determined by some idempotent in $\mathcal{M}(A)$ if and only if $\pi$ is FDS and Ann $(\operatorname{ker}(\pi)) \neq\{0\}$.

Proof. The "only if" part of the assertion is trivial. We prove the other direction. To this end assume that $\pi$ is FDS and Ann $(\operatorname{ker}(\pi)) \neq\{0\}$. By (17, Prop. 4.2.1.5 (iii)) there exists a subspace $H_{0} \subset H$ such that $\pi(A)$ acts algebraically irreducibly on $H_{0}$. Then the argument in $(13$, p. 65$)$ shows that $\pi(A)$ contains self-adjoint minimal idempotents. Let $K=\operatorname{ker}(\pi)$ and $M=\operatorname{Ann}(K)$. The set $\pi(M+K)$ is a nonzero ideal of $\pi(A)$, and therefore $\pi(M+K)$ must contain some $F \in \mathcal{M}(\pi(A))$. Choose $f=f^{*} \in M+K$ such that $\pi(f)=F$. Then $f$ has a unique decomposition

$$
f=e+h \text { where } e \in M \text { and } h \in K .
$$

We have $f^{2}=e^{2}+h^{2}$ and $f^{2}-f \in K$. Therefore

$$
e^{2}-e=\left(f^{2}-f\right)+\left(h-h^{2}\right) \in K \text {. }
$$

Thus $e=e^{2}$, and a similar argument shows $e=e^{*}$.

If $g \in A$, then there exists a scalar $\lambda$ such that $F \pi(g) F=\lambda F$. Therefore $f g f-\lambda f \in K$, and

$$
e g e-\lambda e=(f g f-\lambda f)+(\lambda h-h g h) \in K
$$

Thus $e g e=\lambda e$. Finally, note that $\pi(e) \neq 0$, so that $\pi$ is determined by $e$.

Taken together, Lemmas 1, 2 and 3 prove Theorem 1.

\section{Conditions that imply that a square integrable representation is integrable}

In (4) J. Dixmier gives an example of a square integrable representation which is not integrable. Nevertheless, there are large classes of unimodular LC groups with the property that every irreducible square integrable representation is integrable. The aim of 
this section is to establish this for $I N$-groups $G$ which are either hermitian ( $G \in[H E R])$ or have polynomial growth $(G \in[P G])$ and for almost connected nilpotent groups. One main ingredient in the proof is the work of $T$. Sund in (14). Another is the next proposition.

We need some additional notation. Let $A$ be an $A^{*}$-algebra. We denote the spectrum in $A$ of $f \in A$ by sp $(f ; A)$. The algebra $A$ is called hermitian if for every $f=f^{*} \in A$, $\operatorname{sp}(f ; A)$ is real (a group $G$ is hermitian if $L^{1}(G)$ is hermitian). Now assume that $A$ is an $A^{*}$-algebra. Then $A$ has a largest $C^{*}$-norm $\gamma$. We denote by $\bar{A}$ the completion of $A$ with

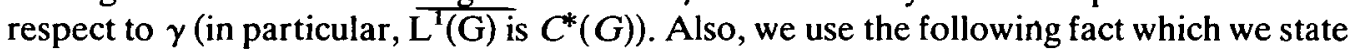
without proof.

Lemma 4. Let $A$ be a hermitian $A^{*}$-algebra. Then

$$
\operatorname{sp}(f ; A)=\operatorname{sp}(f ; \bar{A}) \text { forall } f=f^{*} \in A .
$$

Proposition 3. Let $A$ be a hermitian $A^{*}$-algebra. If $e \in M(\bar{A})$, then there exists $f \in M(A)$ such that the restriction to $A$ of the representation of $\bar{A}$ determined by $e$ is determined by $f$.

Proof. Let $e \in M(\bar{A})$. By definition, $\bar{A} e$ with the appropriate inner product is a dense subspace of the representation space of the representation of $\bar{A}$ determined by $e$. Then to prove the proposition it suffices by Proposition 1 to find $f \in \mathcal{M}(A)$ such that $f \bar{A} e \neq\{0\}$. This we proceed to do.

Now let

$$
B_{k}=\left\{z \in C ;|z-k|<\frac{1}{4}\right\}, \quad k=0,1 .
$$

Using the upper semi-continuity of the spectrum and Lemma 4, we can choose $g=g^{*} \in A$ with $\gamma(g-e)<\frac{1}{4}$ and $\operatorname{sp}(g ; A) \subset B_{0} \cup B_{1}$. Let $M$ be a maximal commutative *-subalgebra containing $g$. Using the analytic functional calculus (with the analytic function which is 1 on $B_{1}$ and 0 on $B_{0}$ ), choose $f \in M$ with

$$
\hat{f} \equiv 0 \quad \text { on }(\hat{g})^{-1}\left(B_{0}\right) \text { and } \hat{f} \equiv 1 \text { on }(\hat{g})^{-1}\left(B_{1}\right)
$$

Then $f$ is a self-adjoint idempotent in $M$. By Lemma 4 and (13, Lemma (4.8.1)) we have for all $h=h^{*} \in M$

$$
\gamma(h)=\sup \{|\lambda|: \lambda \in \operatorname{sp}(h ; A)\}=\sup \{|\lambda|: \lambda \in \operatorname{sp}(h ; M)\} .
$$

It follows that $\gamma(g-f) \leqq \frac{1}{4}$, and thus $\gamma(e-f)<\frac{1}{2}$. Define projection operators $E$ and $F$ on the Banach space $\bar{A}$ by

$$
E(g)=e g e \quad \text { and } \quad F(g)=f g f \quad(g \in \bar{A}) .
$$

Then for all $g \in \bar{A}$,

$$
\begin{aligned}
\gamma((E-F) g) & =\gamma(e g e-f g f) \\
& \leqq \gamma((e-f) g e)+\gamma(f g(e-f)) \\
& \leqq \gamma(e-f) \gamma(g)+\gamma(g) \gamma(e-f)
\end{aligned}
$$

Therefore the norm of $E-F$ as an operator on $\bar{A}$ is less than one. It follows by (6, 
Corollary 2.6, p. 200) that the dimension of the range of $F$ is the same as the dimension of the range of $E$ which is one. Thus $f \in \mathcal{M}(A)$. If $f \bar{A} e=\{0\}$, then

$$
1=\gamma(e)=\gamma((e-f) e) \leqq \gamma(e-f)<\frac{1}{2} .
$$

This completes the proof.

Let $G \in[P G]$ (note: any nilpotent group is $[P G]$ by $(\mathbf{1 0}, \mathrm{p} .71)$ ). Assume in addition that $G$ is compactly generated. Then T. Pytlik has shown that there is a polynomial weight $\omega(x)$ on $G$ such that $L_{\omega}^{1}(G)$, the corresponding convolution algebra of integrable functions with respect to $\omega(x) d x$, is a hermitian Banach *algebra (11, Corollary 7). Let $r_{\omega}$ and $\gamma_{\omega}$ denote the spectral radius and largest $C^{*}$-norm on $L_{\omega}^{1}(G)$, respectively. Let $r$ and $\gamma$ be the corresponding functions on $L^{\mathbf{1}}(G)$.

Lemma 5. For all $\in L_{\omega}^{1}(G), \gamma_{\omega}(f)=\gamma(f)$. Thus the completion of $L_{\omega}^{1}(G)$ with respect to $\gamma_{\omega}$ is identified with $C^{*}(G)$.

Proof. Consider the three equalities

$\begin{array}{lll}\text { (1) }\|\rho(f)\|=\gamma(f) & \text { for } f=f^{*} \in L^{1}(G) \text {; } \\ \text { (2) } r_{\omega}(f)=\|\rho(f)\| & \text { for } f=f^{*} \in L^{1}(G) \text {; } \\ \text { (3) } \gamma_{\omega}(f)=r_{\omega}\left(f^{*} f\right)^{1 / 2} & \text { for } f \in L_{\omega}^{1}(G) .\end{array}$

The equality in (1) follows from the fact that $G$ is amenable (10, p. 75) and (5, p. 63); (2) is (11, Theorem 6); and (3) follows from the result that $L_{\omega}^{1}(G)$ is hermitian and (9, Theorem, p. 523). Combining (1), (2) and (3) we have

$$
\gamma_{\omega}(f)=\gamma(f) \text { for all } f=f^{*} \in L_{\omega}^{1}(G)
$$

This extends to all $f \in L_{\boldsymbol{\omega}}^{\mathbf{1}}(G)$ since

$$
\gamma_{\omega}(f)^{2}=\gamma_{\omega}\left(f^{*} f\right)=\gamma\left(f^{*} f\right)=\gamma(f)^{2} .
$$

Remark. Lemma 5 implies that the *-representations of $L^{1}(G)$ are essentially the same as those of the Beurling algebra $L_{\omega}^{1}(G)$. Of course it is automatic that a (cyclic, irreducible) *-representation of $L^{1}(G)$ determines a (cyclic, irreducible) *-representation of $L_{\omega}^{1}(G)$ by restriction. But also if $(\pi, H)$ is a ${ }^{*}$-representation of $L_{\omega}^{1}(G)$, then by Lemma 5

$$
\gamma(f)=\gamma_{\omega}(f) \geqq \pi(f) \quad\left(f \in L_{\omega}^{1}(G)\right) .
$$

Then $\pi$ lifts to a ${ }^{*}$-representation of $L^{1}(G)$ by continuity.

Theorem 2. Let $G$ be a unimodular, $\sigma$-compact, LC group. Assume that either

(i) $G \in[H E R]$, or

(ii) $G$ is compactly generated and $G \in[P G]$.

Then the following are equivalent for $\pi \in \operatorname{Irr}(G)$ :

(1) $\{\pi\}$ is open in $\hat{G}$;

(2) $\pi$ is integrable;

(3) $\pi$ is determined by some $f \in \mathcal{M}\left(L^{1}(G)\right)$. 
Proof. By Theorem 1, (2) and (3) are equivalent for any unimodular group. Also (3) implies (1) by Proposition 2 .

Now assume that $G$ is $\sigma$-compact and $G \in[H E R]$. If $\{\pi\}$ is open in $\hat{G}$, then $\pi$ is determined by some idempotent $e \in \mathcal{M}\left(C^{*}(G)\right)$ by Proposition 2. But then by Proposition $3 \pi$ is determined by some idempotent $f \in \mathcal{M}\left(L^{1}(G)\right)$. This completes the proof in this case.

When (ii) holds, then Lemma 5 implies that $C^{*}(G)$ and $\overline{L_{\omega}^{1}(G)}$ (using the notation of Proposition 3) are completely identified. Therefore, arguing as above, we have that when $\{\pi\}$ is open in $\hat{G}$, then $\pi$ restricted to $L_{\omega}^{1}(G)$ is determined by some $f \in M\left(L_{\omega}^{1}(G)\right)$. Since $f \in \mathcal{M}\left(L^{1}(G)\right)$ as well, the result follows.

Concerning Theorem 2, it seems to be an open question whether (ii) implies (i). J. Ludwig has proved in (19) that if $G$ is connected and $G \in[P G]$, then $G$ is hermitian. On the other hand there is an example of a $\sigma$-compact group $G \in[P G]$ such that $G \notin[H E R]$; see (18).

Assume that $\pi$ is a unitary representation of a unimodular $L C$ group $G$. Assume that $K$ is a compact normal subgroup of $G$ contained in the kernel of $\pi$. Define $\dot{\pi}$ on $G / K$ by

$$
\dot{\pi}(x K)=\pi(x) \quad(x \in G) .
$$

It is easy to check using Weil's formula (12, p. 70) that $\pi$ is square integrable (integrable) on $G$ if and only if $\dot{\pi}$ is square integrable (integrable) on $G / K$.

Theorem 3. Let G be a LC group which is in addition at least one of the following:

(i) a compactly generated nilpotent Lie group;

(ii) a compactly generated almost connected nilpotent group;

(iii) a $\sigma$-compact hermitian IN-group;

(iv) an almost connected IN-group.

Then for $\pi \in \operatorname{Irr}(G)$ the following are equivalent:

(1) $\pi$ is square integrable;

(2) $\pi$ is integrable;

(3) $\{\pi\}$ is open in $G$.

Proof. As we noted earlier, any nilpotent group is in $[P G](\mathbf{1 0}$, p. 71). Also, an almost connected IN-group is hermitian (10, p. 68). Thus in each case (i)-(iv) G is either compactly generated and in $[P G]$ or $G$ is hermitian. Therefore a direct application of Theorem 2 yields the equivalence of (2) and (3). T. Sund proves in (14) that if $G$ is either an $I N$-group or a nilpotent Lie group, then every square integrable irreducible representation $\pi$ of $G$ has $\{\pi\}$ open in $\hat{G}$. This proves the result in all cases except (ii).

To prove this last case, assume that $G$ is an almost connected nilpotent group and $\pi \in \operatorname{Irr}(G)$ is square integrable. By $(8$, p. 517) there exists a compact normal subgroup $K$ of $G$ such that $K \subset \operatorname{ker}(\pi)$ and $G / K$ is a nilpotent Lie group. Applying the remarks preceding the theorem, the representation $\dot{\pi}$ on $s G / K$ determined by $\pi$ is square integrable, and thus integrable by a previous argument. Therefore $\pi$ is integrable. 


\title{
REFERENCES
}

(1) B. A. BARNES, Locally separable Banach $*$-algebras: preprint.

(2) C. W. CURTIS and I. REINER, Representation Theory of Finite Groups and Associative Algebras (Interscience Publishers, John Wiley \& Sons, New York, 1966).

(3) J. DiXmier, Les C $C^{*}$-algebres et leurs Representations (Gautier-Villars, Paris, 1969).

(4) J. Dixmier, Points isoles dans le dual d'un groupe localement compact, Bull. Sc. Math. 85 (1961), 91-96.

(5) F. P. Greenleaf, Invariant Means on Topological Groups (Van Nostrand, New York, 1969).

(6) T. Kato, Pertubation Theory for Linear Operators (Springer-Verlag, New York, 1966).

(7) R. L. LiPsMAN, Dual topology for principal and discrete series, Trans. Amer. Math. Soc. 152 (1970), 399-417.

(8) D. MiliceC, Representations of almost connected groups, Proc. Amer. Math. Soc. 47 (1975), 517-518.

(9) T. W. Palmer, Hermitian Banach *-algebras, Bull. Amer. Math. Soc. 78 (1972), 522-524.

(10) T. W. PALMER, Classes of nonabelian, noncompact, locally compact groups, Rocky Mountain J. of Math. 8 (1978), 683-741.

(11) T. PYTLIK, On the spectral radius of elements in group algebras, Bull. de l'Academie Polonaise des Sci., Serie des sci. math., astron., et phys. 21 (1973), 899-902.

(12) H. ReITER, Classical Harmonic Analysis and Locally Compact Groups (Oxford Univ. Press, London, 1968).

(13) C. E. RicKART, Banach Algebras (D. Van Nostrand, Princeton, N.J., 1960).

(14) T. SUND, Isolated points in duals of certain locally compact groups, Math. Ann.224 (1976), 33-39.

(15) P. S. WANG, On integrable representations, Math. Z. 147 (1976), 201-203.

(16) P. S. WANG, On isolated points in the dual spaces of locally compact groups, Math. Ann. 218 (1975), 19-34.

(17) G. WARner, Harmonic Analysis on Semi-simple Lie Groups, Vol. I (Springer-Verlag, Berlin, 1972).

(18) J. Fountain, R. RAmSEy and J. H. Williamson, Functions of measures on compact groups, Proc. Royal Irish Acad. Sect. A 76 (1976), 231-251.

(19) J. LuDwIG, A class of symmetric and a class of Wiener group algebras, J. Functional Analysis 31 (1979), 187-194.

(20) M. RIEFFEL, Square integrable representations of Hilbert algebras, J. Functional Analysis 3 (1969), 265-300.

\author{
UNIVERSTTY OF OREGON \\ EUGENE, OREGON 97403 \\ U.S.A.
}

